\title{
Obesity and morbid obesity associated with higher odds of hypoalbuminemia in adults without liver disease or renal failure
}

This article was published in the following Dove Press journal: Diabetes, Metabolic Syndrome and Obesity:Targets and Therapy 8 November 2017

Number of times this article has been viewed

\author{
Rana H Mosli' \\ Hala H Mosli² \\ 'Clinical Nutrition Department, \\ Faculty of Applied Medical Sciences, \\ King Abdulaziz University, Jeddah, \\ Kingdom of Saudi Arabia; ${ }^{2}$ Division \\ of Endocrinology and Metabolism, \\ Department of Medicine, Faculty of \\ Medicine, King Abdulaziz University, \\ Jeddah, Kingdom of Saudi Arabia
}

Background and objective: Studies are needed in order to inform recommendations for interpreting albumin levels among obese individuals without known medical conditions associated with hypoalbuminemia. The objective of this study was to examine the association of obese and morbidly obese status with hypoalbuminemia, while adjusting for age, sex, diabetes, prediabetes, diabetic nephropathy, and nephrotic syndrome.

Patients and methods: Retrospective data collection from adult patients presenting to the outpatient Endocrinology and Metabolism Clinic was performed between January 2015 and December 2015. An initial sample of 180 patients was selected. After excluding patients who were younger than 18 years, who had known cases of liver disease or renal failure, or who had missing data, a final sample of 122 subjects was identified. Serum albumin and objectively measured weight and height data were retrieved from hospital records. A board-certified endocrinologist reviewed patient records to identify the presence of renal and diabetic conditions. Descriptive statistics were used to examine sample characteristics. Multiple logistic regression analysis was used to examine the association of obesity and morbid obesity with hypoalbuminemia (serum albumin $<34 \mathrm{~g} / \mathrm{L}$ ) while adjusting for age, sex, diabetes, prediabetes, diabetic nephropathy, and nephrotic syndrome.

Results: Approximately $43 \%$ of the sample were categorized as obese and $13 \%$ were categorized as morbidly obese. The mean serum albumin level was $38.00 \mathrm{~g} / \mathrm{L}$ (standard deviation $[\mathrm{SD}]=4.26)$ among subjects who were neither overweight nor obese, $38.35 \mathrm{~g} / \mathrm{L}(\mathrm{SD}=0.48)$ among overweight subjects, $34.57 \mathrm{~g} / \mathrm{L}(\mathrm{SD}=4.71)$ among obese subjects, and $33.81 \mathrm{~g} / \mathrm{L}(\mathrm{SD}=$ 3.71) among morbidly obese subjects. Adjusting for age, sex, diabetes, prediabetes, nephrotic syndrome, and diabetic nephropathy, obese subjects had significantly higher odds of hypoalbuminemia (odds ratio [OR]: 4.10, 95\% confidence interval [CI]: $1.50-11.27, P$-value $=0.006$ ), as did morbidly obese subjects (OR: $6.94,95 \% \mathrm{CI}: 1.91-25.23, P$-value $=0.003$ ).

Conclusion: The findings suggest that obesity and morbid obesity can be considered as independent predictors of hypoalbuminemia. The findings can be used to inform future studies aiming to better understand the association of obesity and morbid obesity with hypoalbuminemia and to help inform guidelines for clinicians on how to correctly interpret and utilize serum albumin data for obese individuals.

Keywords: obesity, morbid obesity, albumin, hypoalbuminemia, inflammation, diabetes mellitus

\section{Introduction}

Obesity, defined as excessive fat accumulation in the body, ${ }^{1}$ continues to be a major global concern. ${ }^{2}$ As of 2014, approximately half (52\%) of the adults in the world are overweight or obese. ${ }^{3}$ Overconsumption of calories and a sedentary lifestyle are
Clinical Nutrition Department, Faculty of Applied Medical Sciences, King Abdulaziz University, PO Box 80215, Jeddah 21589, Kingdom of Saudi Arabia

Email aimuwseli@kau.edu.sa 
contributing factors to the uprise of this epidemic. ${ }^{2}$ Owing to the nutrition transition and rapid shifts seen in dietary and physical activity patterns in most parts of the worlds, obesity is now a health condition that is highly prevalent in both developing and industrialized countries. ${ }^{4}$ Obesity leads to an increased likelihood of developing metabolic syndrome and an array of serious diseases, including type 2 diabetes, cardiovascular disease, nonalcoholic fatty liver disease, as well as colon, breast and other hormone-related cancers., ${ }^{5,6}$

Treatment of obesity requires a multidisciplinary team approach. In order to effectively prevent and treat complications while initiating weight loss and modifying lifestyle behaviors, physicians, nurses, dietetic specialists, psychologists and social workers must be collaboratively involved in patient management. ${ }^{7}$ As part of the nutrition care process, clinical dietitians usually perform a biochemical assessment in which laboratory data are interpreted and correlated with the nutrition-related problem and signs and symptoms. However, guidelines for interpreting biochemical data for the obese patients are not fully established. Specifically, there is a lack of published studies that inform recommendations for interpreting albumin laboratory values among the obese patients.

Albumin, the most abundant plasma protein, has been traditionally used as an indicator for malnutrition and chronic starvation. ${ }^{8}$ Although the Academy of Nutrition and Dietetics urges the need to interpret albumin values with caution, due to its likelihood to decrease with kidney and inflammatory diseases, ${ }^{8}$ instructions for interpreting abnormal albumin levels among obese patients are not clearly stated. Evidence suggests that obesity is a state of chronic inflammation in which hypertrophy of adipocytes and hypoxia lead to the production of proinflammatory cytokines, such as tumor necrosis factor alpha. ${ }^{9,10}$ It is plausible that this state of inflammation may lead to altered serum albumin levels among the obese.

Although one study found that serum albumin is low among obese and nondiabetic children, ${ }^{11}$ others examined the correlation between body mass index (BMI) and albumin among diabetic adults ${ }^{12,13}$ or the correlation between BMI and glycated albumin; ${ }^{14}$ we were unable to identify any studies examining obesity and morbid obesity as independent predictors of hypoalbuminemia among adults. Such studies are needed in order to inform recommendations for interpreting albumin among obese individuals. The goal of this study was to examine the association of obese status and morbidly obese status with hypoalbuminemia, while adjusting for potential confounders, including age, sex, diabetes, prediabetes, diabetic nephropathy and nephrotic syndrome.

\section{Patients and methods}

\section{Sample and procedure}

Retrospective data were collected from all patients presenting to the outpatient Endocrinology and Metabolism Clinic between January 2015 and December 2015. An initial sample was identified for a study involving vitamin D serum levels. Thus, the original sample included 180 patients with complete serum vitamin D data. For the purpose of the current study, we selected patients who were older than 18 years with available albumin and weight and height data. Patients with known cases of liver disease or renal failure (with a diagnosis documented in previous notes or those with abnormal abdominal examination, abnormal liver enzymes or abnormal international normalized ratio [INR]) were excluded from the dataset. A final sample of 122 subjects was identified. The sample included in this analysis $(\mathrm{n}=122)$ did not differ from the sample not included $(\mathrm{n}=58)$ with regard to age, sex and nationality $(P$-values $>0.1)$. Ethical approval to perform the study was obtained from the Unit of Biochemical Ethics at King Abdulaziz University. Patients' identities were protected by linking each patient's data to an anonymous identification number and eliminating names and contact information. Patient informed consent requirement was waived by the unit, given the retrospective nature of the study.

Using a standardized data collection form, a registered nurse who was part of the research team and was trained in using the hospital's electronic server extracted the following data from patients' electronic medical files: patient age, sex, nationality, weight and height and serum albumin level (Siemens Dimension Vista ${ }^{\circledR}$ System Flex ${ }^{\circledR}$ reagent cartridge by spectrophotometry). Weight and height were objectively measured by a trained hospital staff at the time of visit using standardized procedures, and BMI was automatically calculated (as weight in $\mathrm{kg} /$ height in $\mathrm{m}^{2}$ ) and recorded in the patients' electronic medical record. To extract data on the presence of renal conditions and diabetes, files were reviewed by one of the principal investigators (who was a board-certified endocrinologist) for renal function and presence of diabetes mellitus or prediabetes and nephrotic syndrome; data were recorded in the data collection form. All recorded data were collected from each patient within the same 1-month window.

\section{Measures}

Primary outcome: hypoalbuminemia

As widely employed, we defined hypoalbuminemia as a serum albumin level $<34 \mathrm{~g} / \mathrm{L} .{ }^{15}$ 
Primary predictor: obese and morbidly obese status Owing to ease of use and correlation with body fat, ${ }^{1}$ we used BMI to categorize weight status. Obese status was defined as BMI $\geq 30 \mathrm{~kg} / \mathrm{m}^{2} .^{1}$ Morbidly obese status was defined as BMI $\geq 40 \mathrm{~kg} / \mathrm{m}^{2}{ }^{16}$

\section{Covariates}

We identified variables to which to adjust in our statistical analysis a priori from the literature. Increase in age was previously found to be associated with decrease in serum albumin level, ${ }^{17}$ and increase in age was found to be associated with increase in BMI. ${ }^{18}$ Similarly, sex was previously found to be associated with serum albumin, ${ }^{17}$ as well as weight status. ${ }^{16}$ Thus, for use as covariates in statistical analyses, age was calculated based on the patient's date of birth and date of data collection, and patient's sex was retrieved from the electronic medical file.

Serum albumin is considered to be a negative acute-phase reactant. Negative acute-phase reactants are proteins that decrease with inflammation, due to decreased liver synthesis probably as a consequence of increased hepatic synthesis of positive acute-phase reactants. ${ }^{19,20}$ Therefore, serum albumin level is known to be affected by certain health conditions, most commonly, renal conditions and diabetes. Renal conditions and diabetes are also associated with weight status, such that obese individuals have been found to be at an increased risk for developing these health complications. ${ }^{5,21}$ Thus, to examine obesity and morbid obesity as independent predictors of hypoalbuminemia, data on presence of renal conditions and diabetes were retrieved. Subjects were determined to have diabetes mellitus if glycosylated hemoglobin (HbA1c) was $\geq 6.5 \%$, fasting blood sugar was $\geq 7.0 \mathrm{mmol} / \mathrm{L}$, random blood sugar was $\geq 11.1 \mathrm{mmol} / \mathrm{L}$ or a $75 \mathrm{~g}$ oral glucose tolerance test (OGTT) with a 2 -hour reading was $\geq 200 \mathrm{mmol} / \mathrm{L}$. Subjects were also deemed diabetic if he/she was on treatment for diabetes using oral hypoglycemic agents or insulin as recorded in the electronic medical record. Prediabetes was diagnosed if the patient had untreated $\mathrm{HbAlc}$ of $5.9-6.49 \%$, fasting blood sugar of $6.1-6.9 \mathrm{mmol} / \mathrm{L}$, random blood sugar or 2-hour post OGTT of $7.8-11 \mathrm{mmol} / \mathrm{L}^{22,23}$ Diabetic nephropathy was diagnosed if a patient with diabetes or prediabetes had an urine microalbumin/creatinine ratio above local lab cutoff of $30 \mathrm{mg} / \mathrm{g}$. Nephrotic syndrome could not be diagnosed using urine microalbumin/creatinine ratio. However, if nephrotic syndrome was suspected based on markedly elevated urine albumin/creatinine ratio (ACR), the patient was further evaluated for elevated low-density lipoprotein (LDL) and total cholesterol and 24-hour urine albumin $>3 \mathrm{~g} /$ day. Clinical notes were reviewed for documentation of anasarca.

\section{Statistical analysis}

Statistical analyses were conducted using IBM SPSS Statistics 21.0 (IBM Corporation, Armonk, NY, USA). Descriptive statistics were used to describe characteristics of the total sample.

We conducted logistic regression to examine the association of obesity and morbid obesity status (primary predictors) with hypoalbuminemia (primary outcome). We first ran an unadjusted regression model. Then, we ran multiple logistic regression models while adjusting for potential confounders, including age, sex, diabetes, prediabetes, diabetic nephropathy and nephrotic syndrome.

Additionally, in order to further investigate whether the correlation between weight status and hypoalbuminemia is limited only to obese and morbidly obese subjects or whether it also exists among subjects who were not obese or morbidly obese, we tested a logistic regression model with BMI as the predictor and hypoalbuminemia as the outcome in a dataset comprising only subjects whose BMI was $<30 \mathrm{~kg}$ / $\mathrm{m}^{2}$. The original dataset did not include any subjects who were underweight (BMI $\left.<18.5 \mathrm{~kg} / \mathrm{m}^{2}\right)$. Thus, the selected subjects for this additional analysis $(\mathrm{n}=62)$ were all either overweight $\left(25 \mathrm{~kg} / \mathrm{m}^{2} \leq \mathrm{BMI}<30 \mathrm{~kg} / \mathrm{m}^{2}\right)$ or within the normal range $\left(18.5 \mathrm{~kg} / \mathrm{m}^{2} \leq \mathrm{BMI}<25 \mathrm{~kg} / \mathrm{m}^{2}\right)$. An unadjusted model was first tested, and then the model was adjusted for age, sex, diabetes, prediabetes, diabetic nephropathy and nephrotic syndrome.

\section{Results}

More than half of the study sample $(62 \%)$ were Saudi nationals, and the majority of subjects $(77 \%)$ were females. Approximately $43 \%$ of the sample was categorized as obese, and $13 \%$ was categorized as morbidly obese (Table 1 ).

The results of multiple logistic regression, including unadjusted and adjusted odds ratios (ORs), are shown in Table 2. Obese and morbidly obese subjects had significantly higher odds of hypoalbuminemia (OR: $3.79,95 \%$ confidence interval (CI): $1.66-8.76$ and OR: $4.64,95 \%$ CI: $1.55-13.95$, respectively). These associations were not weakened by inclusion of potential confounders in the regression models; adjusting for age, sex, diabetes, prediabetes, nephrotic syndrome and diabetic nephropathy, obese subjects had significantly higher odds of hypoalbuminemia (OR: 4.10, 95\% CI: $1.50-11.27$ ), as did morbidly obese subjects (OR: 6.94, 95\% CI: 1.91-25.23). 
Table I Sample characteristics

\begin{tabular}{|c|c|}
\hline Variable & Total $(n=122)$ \\
\hline \multicolumn{2}{|l|}{ Nationality, n (\%) } \\
\hline Saudi & $75(61.5)$ \\
\hline Non-Saudi & $47(38.5)$ \\
\hline \multicolumn{2}{|l|}{ Sex, n (\%) } \\
\hline Male & $27(22.1)$ \\
\hline Female & $95(77.9)$ \\
\hline Age, $M(S D)$ & $50.11(15.48)$ \\
\hline BMI, M (SD) & $30.91(8.15)$ \\
\hline Obese, n (\%) & $60(49.2)$ \\
\hline Morbidly obese, n (\%) & $16(13.1)$ \\
\hline \multicolumn{2}{|l|}{ Albumin level, $M(S D)$} \\
\hline Total sample & $36.24(4.69)$ \\
\hline Not overweight or obese & $38.00(4.26)$ \\
\hline Overweight & $38.35(4.08)$ \\
\hline Obese & $34.57(4.7 I)$ \\
\hline Morbidly obese & $33.8 I(3.7 I)$ \\
\hline \multicolumn{2}{|c|}{ Low albumin level (<34 g/L), n (\%) } \\
\hline Yes & $38(31.1)$ \\
\hline No & $84(68.9)$ \\
\hline
\end{tabular}

Note: The table shows $M$ and SD values or counts $(n)$ and percentages (\%). Abbreviations: M, mean; SD, standard deviation; BMI, body mass index.

Table 2 Unadjusted and adjusted associations of obesity and morbid obesity with hypoalbuminemia $(n=122)$

\begin{tabular}{|c|c|c|}
\hline Primary predictors & $\begin{array}{l}\text { Unadjusted OR } \\
(95 \% \mathrm{Cl})\end{array}$ & $\begin{array}{l}\text { Adjusted OR } \\
(95 \% \mathrm{Cl})^{\mathrm{a}}\end{array}$ \\
\hline \multicolumn{3}{|l|}{ Obesity status } \\
\hline Obese & $3.79(1.66-8.76)^{* *}$ & $4.10(1.50-11.27)^{* *}$ \\
\hline Not obese & I & I \\
\hline Adjusted $R^{2}$ & 0.12 & 0.28 \\
\hline \multicolumn{3}{|l|}{ Morbid obesity status } \\
\hline Morbidly obese & $4.64(1.55-13.95)^{* *}$ & $6.94(1.91-25.23)^{* *}$ \\
\hline Not morbidly obese & I & I \\
\hline Adjusted $R^{2}$ & 0.10 & 0.29 \\
\hline
\end{tabular}

Notes: Hypoalbuminemia is defined as serum albumin $<34 \mathrm{~g} / \mathrm{L}$, obese status is defined as $B M I \geq 30 \mathrm{~kg} / \mathrm{m}^{2}$ and morbidly obese status is defined as $B M I \geq 40 \mathrm{~kg} / \mathrm{m}^{2}$. aModels adjusted for potential confounders: age, sex, diabetes, prediabetes, nephrotic syndrome, and diabetic nephropathy. $* * P$-value $<0.01$.

Abbreviations: $\mathrm{OR}$, odds ratio; $\mathrm{Cl}$, confidence interval; $\mathrm{BMI}$, body mass index.

Further analysis revealed that there was a negative association between BMI and odds of hypoalbuminemia among subjects who were not obese or morbidly obese. Among normal weight and overweight subjects, increase in BMI was associated with lower likelihood of hypoalbuminemia after adjusting for potential confounders. This association, however, only approached statistical significance (OR: 0.77, 95\% CI: $0.59-1.02, P$-value $=0.07$.

\section{Discussion}

We found that obesity and morbid obesity status were each associated with higher odds of hypoalbuminemia. Results were not attenuated by inclusion of age, sex, diabetes, prediabetes, nephrotic syndrome and diabetic nephropathy in the regression models. These findings suggest that obesity and morbid obesity can be considered as independent predictors of hypoalbuminemia. Further analysis also shows that among subjects who were normal weight or overweight, increase in BMI was associated with lower likelihood of hypoalbuminemia. This further supports our hypothesis that hypoalbuminemia may be uniquely associated with the state of excess adiposity (i.e., obesity and morbid obesity). Indeed, lower BMI in subjects who were normal weight or overweight may be associated with some degree of protein malnutrition, which might explain the negative association between BMI and hypoalbuminemia.

Our findings are consistent with findings from previous studies, showing a negative association between obesity and albumin level among children. ${ }^{11}$ Although previous studies identified a negative correlation between BMI and albumin level among adults, these study samples included only diabetic subjects, ${ }^{12,13}$ assessed glycated albumin rather than albumin level ${ }^{14}$ or only compared albumin levels between different obesity classes. ${ }^{24}$ Another study in Brazil that found that the majority $(85 \%)$ of overweight or obese elderly patients who were admitted at the hospital had hypoalbuminemia correlated hypoalbuminemia with nutritional status and age rather than BMI and the presence of metabolic syndrome. ${ }^{25}$ To our knowledge, our study is among the first to report greater likelihood of hypoalbuminemia among obese and morbidly obese adults, while taking into account factors that may contribute to decreased albumin levels, such as age, sex, diabetes, prediabetes, diabetic nephropathy and nephrotic syndrome.

Although the exact mechanism underlying the association of obesity with hypoalbuminemia is unknown, it is plausible that hypoalbuminemia is caused by the physiologic changes accompanied by the inflammatory state seen in obesity and morbid obesity. Future studies are needed to identify the exact mechanism by which obesity and morbid obesity lead to hypoalbuminemia.

Serum albumin levels can be used as a prognostic tool that helps predict patient medical and surgical outcomes. ${ }^{15,20,26}$ The association of low albumin with adverse patient outcomes may be due to the fact that albumin is a biomarker for severe protein malnutrition. ${ }^{20}$ However, albumin was found to be an independent predictor of morbidity and mortality in critically ill patients. ${ }^{27}$ Surgical data found that hypoalbuminemia detected preoperatively was usually associated with adverse outcomes, specifically delayed wound healing, poor surgical outcome, need for repeat surgery and higher readmission 
rates. ${ }^{28}$ While these outcomes were all thought to be related to the presumed underlying malnutrition manifesting in the form of low albumin, the exact mechanism is not yet known in detail. Obesity state is being increasingly recognized as a state of chronic inflammation. However, it is unclear if this chronic low-grade inflammation is part of the underlying cause of the effects of hypoalbuminemia on the surgical outcomes outlined earlier. ${ }^{29}$ The impact of low albumin level on overall health and well-being among the obese is unknown. Future studies are needed in order to examine the effect of hypoalbuminemia on health outcomes among obese and morbidly obese individuals.

This study has several strengths. We explored the association of obesity and morbid obesity with hypoalbuminemia, while carefully accounting for some of the most important conditions that may contribute to low albumin levels using the expertise of a board-certified endocrinologist. Furthermore, weight and height of subjects were objectively measured by trained hospital staff using standardized procedures. This study also has some limitations. Our sample size was relatively small. Studies with larger sample sizes are needed to confirm our findings. There might have been residual confounding by factors that we did not account for in our analysis (e.g., subclinical health conditions or an undetected liver disease), and we did not obtain data on subjects' dietary intake. Future studies can include comprehensive interviews with subjects in order to obtain detailed information on factors that may contribute to hypoalbuminemia.

\section{Conclusion}

Our findings can help inform future studies aiming to better understand the association of obesity and morbid obesity with hypoalbuminemia. Findings from our study can help inform guidelines for clinical dietitians on how to correctly interpret and utilize serum albumin data for obese individuals. Given the increasing rates of obesity in both industrialized and developing countries, ${ }^{4}$ a clear consensus needs to be established among the international community regarding how to assess and interpret biochemical data for the obese in order to optimize obesity management and efficiently employ the nutrition care process.

\section{Author contributions}

RHM designed the study, analyzed the data and drafted the initial manuscript. HHM coordinated and supervised data collection, provided input on the analysis plan and critically reviewed the manuscript. Both authors contributed toward data analysis, drafting and critically revising the paper, gave final approval of the version to be published, and agree to be accountable for all aspects of the work.

\section{Disclosure}

The authors report no conflicts of interest in this work.

\section{References}

1. Romero-Corral A, VK VKS, Sierra-Johnson J, et al. Accuracy of body mass index in diagnosing obesity in the adult general population. Int $J$ Obes. 2008;32(6):959-966.

2. Rössner S. Obesity: the disease of the twenty-first century. Int J Obes. 2002;26(suppl 4):S2-S4.

3. World Health Organization. Obesity and Overweight [Fact Sheet; updated October 2017]. Available from: http://www.who.int/mediacentre/factsheets/fs311/en/. Accessed August 24, 2017.

4. Popkin BM, Adair LS, Ng SW. Global nutrition transition and the pandemic of obesity in developing countries. Nutr Rev. 2012;70(1): $3-21$.

5. Must A, Spadano J, Coakley EH, Field AE, Colditz G, Dietz WH. The disease burden associated with overweight and obesity. JAMA. 1999;282(16):1523-1529.

6. Bianchini F, Kaaks R, Vainio H. Overweight, obesity, and cancer risk. Lancet Oncol. 2002;3(9):565-574.

7. Thompson WG, Cook DA, Clark MM, Bardia A, Levine JA. Treatment of obesity. Mayo Clin Proc. 2007;82(1):93-102.

8. White JV, Guenter P, Jensen G, et al. Consensus statement of the Academy of Nutrition and Dietetics/American Society for Parenteral and Enteral Nutrition: characteristics recommended for the identification and documentation of adult malnutrition (undernutrition). JAcad Nutr Diet. 2012;112(5):730-738.

9. Sikaris KA. The clinical biochemistry of obesity. Clin Biochem Rev. 2004;25(3):165-182.

10. Netzer N, Gatterer H, Faulhaber M, Burtscher M, Pramsohler S, Pesta D Hypoxia, oxidative stress and fat. Biomolecules. 2015;5(2):1143-1150.

11. Nishimura R, Kanda A, Sano H, et al. Glycated albumin is low in obese, non-diabetic children. Diabetes Res Clin Pract. 2006;71(3): 334-338.

12. Koga M, Matsumoto S, Saito H, Kasayama S. Body mass index negatively influences glycated albumin, but not glycated hemoglobin, in diabetic patients. Endocr J. 2006;53(3):387-391.

13. Miyashita Y, Nishimura R, Morimoto A, Matsudaira T, Sano H, Tajima N. Glycated albumin is low in obese, type 2 diabetic patients. Diabetes Res Clin Pract. 2007;78(1):51-55.

14. Koga M, Otsuki M, Matsumoto S, Saito H, Mukai M, Kasayama S. Negative association of obesity and its related chronic inflammation with serum glycated albumin but not glycated hemoglobin levels. Clin Chim Acta. 2007;378(1):48-52.

15. Herrmann FR, Safran C, Levkoff SE, Minaker KL. Serum albumin level on admission as a predictor of death, length of stay, and readmission. Arch Intern Med. 1992;152:125-130.

16. MacDonald KG. Overview of the epidemiology of obesity and the early history of procedures to remedy morbid obesity. Arch Surg. 2003;138(4):357-360.

17. Ikuko GO, Fukushima H, Shiraki M, et al. Relationship between serum albumin level and aging in community-dwelling self-supported elderly population. J Nutr Sci Vitaminol. 2007;53(1):37-42.

18. Ogden CL, Carroll MD, Kit BK, Flegal KM. Prevalence of obesity and trends in body mass index among US children and adolescents, 1999-2010. JAMA. 2012;307(5):483-490.

19. Jain $S$, Gautam V, Naseem S. Acute-phase proteins: as diagnostic tool. J Pharm Bioallied Sci. 2011;3(1):118.

20. Nicholson JP, Wolmarans MR, Park GR. The role of albumin in critical illness. Br J Anaesth. 2000;85(4):599-610.

21. Chagnac A, Weinstein T, Herman M, Hirsh J, Gafter U, Ori Y. The effects of weight loss on renal function in patients with severe obesity. $J \mathrm{Am}$ Soc Nephrol. 2003;1(14):1480-1486.

22. Cheng AY. Canadian Diabetes Association 2013 clinical practice guidelines for the prevention and management of diabetes in Canada. Introduction. Can J Diabetes. 2013;37:S1-S3. 
23. American Diabetes Association. Diagnosis and classification of diabetes mellitus. Diabetes Care. 2010;33(suppl 1):S62-S69.

24. Fu MC, McLawhorn AS, Padgett DE, Cross MB. Hypoalbuminemia is a better predictor than obesity of complications after total knee arthroplasty: a propensity score-adjusted observational analysis. HSS J. 2017;13(1):66-74.

25. Brock F, Bettinelli LA, Dobner T, Stobbe JC, Pomatti G, Telles CT. Prevalence of hypoalbuminemia and nutritional issues in hospitalized elders. Rev Lat Am Enfermagem. 2016;24:e2736.

26. Dietch ZC, Guidry CA, Davies SW, Sawyer RG. Hypoalbuminemia is disproportionately associated with adverse outcomes in obese elective surgical patients. Surg Obes Relat Dis. 2015;11(4):912-918.
27. Vincent JL, Dubois MJ, Navickis RJ, Wilkes MM. Hypoalbuminemia in acute illness: is there a rationale for intervention? A meta-analysis of cohort studies and controlled trials. Ann Surg. 2003;237(3): 319-334.

28. Meyer CP, Rios-Diaz AJ, Dalela D, et al. The association of hypoalbuminemia with early perioperative outcomes-a comprehensive assessment across 16 major procedures. Am J Surg. 2016;214(5): 871-883.

29. Donath MY, Dalmas É, Sauter NS, Böni-Schnetzler M. Inflammation in obesity and diabetes: islet dysfunction and therapeutic opportunity. Cell Metab. 2013;17(6):860-872.
Diabetes, Metabolic Syndrome and Obesity: Targets and Therapy is an international, peer-reviewed open-access journal committed to the rapid publication of the latest laboratory and clinical findings in the fields of diabetes, metabolic syndrome and obesity research. Original research, review, case reports, hypothesis formation, expert opinion and commentaries are all considered for publication. The manuscript management system is completely online and includes a very quick and fair peer-review system, which is all easy to use. Visit http://www.dovepress.com/testimonials.php to read real quotes from published authors.

Submit your manuscript here: https://www.dovepress.com/diabetes-metabolic-syndrome-and-obesity-targets-and-therapy-journal 